\title{
Wireless Rechargeable Vehicle Based Energy Efficienct Data Transmisison In Wireless Sensor Network
}

\author{
Rajasekar Rangasamy, P.Manikandan, P. Ramkarthik
}

\begin{abstract}
Wireless Energy Transfer technique has attracted increasing attention on authorizing the wireless sensor nodes in recent years. In this paper, we consider a remote battery-powered system where a portable charging vehicle is planned to accuse remote sensor system of hubs' arrangement confinements that may result in less charging efficiency for sensor hubs by charging vehicle. In our method, we used a charging vehicle to charge the nodes whenever needed. Instinctively, there is an unavoidable compromise between the charging distance and the vehicle. For these worries, we go for diminishing the reviving process duration, which contains the voyaging time and energizing time. To this end, we demonstrate that the charging vehicle would go along the briefest way directing. Also, we indicate ideal charging area for every remote charging occurrence.

Keywords: Energy Transfer, Sensor Nodes, Shortest Path, Optimal Charging Location
\end{abstract}

\section{INTRODUCTION}

Vitality effective traffic over the remote sensor organize is developing consistently with expanding number of requesting applications including broadcasting, video meetings, information stream applications and web-content circulations. A large number of these applications require certain rate assurances, and request that the system be used more proficiently than with current ways to deal with fulfill the rate prerequisites. Traffic mapping (stack adjusting) is one specific strategy to do traffic building, which manages the issue of doling out the traffic stack onto pre-set up ways to meet certain prerequisites. Our center is to investigate the impacts of load adjusting the multicast traffic in an intra-space arrange. The current works on multicast directing with power imperatives are allude in the writings. Propose an answer for ideally disseminate the traffic along different multicast trees. Be that as it may, the arrangement covers the situation when there is just a single dynamic source in the system. What's more, it is expected that the angle of an investigative cost work is accessible, which is persistently differentiable and entirely curved. These presumptions may not be sensible because of the dynamic idea of systems.

Despite the fact that they approach the issue under a progressively broad design, common sense of these

Revised Manuscript Received on 14 August, 2019.

Rajasekar Rangasamy, Department of CSE, St.Peter's Engineering College, Hyderabad.

P.Manikandan, Department of CSE, Malla Reddy Engineering College for Women, Hyderabad.

Ramkarthik, Department of CSE, St.Peter's Engineering College, Hyderabad arrangements is restricted because of the unreasonable presumption that the system is lossless as long as the normal connection rates don't surpass the connection limits. Also, a parcel misfortune is in reality substantially more exorbitant when arrange coding is utilized since it possibly influences the translating of an extensive number of different bundles. Furthermore, any factor that changes the min-cut max-stream an incentive between a source and a collector requires the code to be refreshed at each hub at the same time, which brings abnormal state of unpredictability and coordination.

\section{SYSTEM METHODOLOGY}

\section{Power Conservation Protocol}

Power conservation protocols have been proposed for ad hoc multi-hop wireless networks. In topology control methods are studied for two-tiered sensor network architecture. The goal of these works is to reduce the unnecessary energy consumption during the packet delivery process. These protocols are topology oriented and don't consider the geometrical relations. The proposed sleep planning protocol lets each node decide its own sleep schedule so that a certain degree of coverage can be achieved. Moreover, the protocol can achieve differentiated surveillance for certain critical areas within the network. However, the work does not provide a metric on the quality of surveillance; especially for the partially covered eld.GAF and PEAS are two sleep planning protocols that can be used for soft deployment of sensors.

In GAF, sensors use location information to divide the eld into xed square grids. Nodes within a grid switch between sleep and awake, with the guarantee that one node in each grid stays up. The size of the grid can be used to control the density of distribution of active nodes. In PEAS, each node periodically transmits probe messages, and replies to any received probe messages from the neighbor nodes. A node can be of duty if it receives replies for its probes. In this scheme, the density of the active nodes can be controlled by the range of probing messages. In, Scourges et al. studied the trade-of between power conservation and network performance in terms of packet delivery delay. An energy management protocol, STEM, is proposed to work in combination with GAF or SPAN protocols.

\section{Energy saving protocol}

PEAS1, a straightforward convention that can construct a seemingly perpetual sensor arrange and keep up hearty activities utilizing huge 
amounts of affordable, fleeting sensor hubs. PEAS broaden framework working time by keeping just an essential arrangement of sensors working and putting the rest into rest mode. Resting ones wake up once in a while, examining the neighborhood condition and supplanting fizzled ones. The dozing time frames are self-balanced progressively, in order to keep the sensors' wakeup rate generally consistent, accordingly adjusting to high hub densities. PEAS comprises of two basic calculations: Probing Environment and Adaptive Sleeping, which decide (1) which sensors should work and how a wake-up sensor settles on the choice of in the case of returning to rest, and (2) how the normal rest times of sensors are powerfully acclimated to keep a moderately steady wake-up rate, separately.

\section{SYSTEM MODELS}

\section{Topology formation}

In this Phase, Developing Project structure in NS2 should happens. Every hub ought to send hi bundles to its neighbor hub which are in its correspondence range to refresh their topology.

Topology development contains absolutely 39 hubs in condition setup. Every one of the hubs are dynamic hubs which contains same vitality level toward the start.

This paper considered that,

Node 0 - Base Station

Node 38- Mobility Node (WCV)

Node 39 - Service station

\section{Cluster Head Selection}

This phase, forming a cluster head per every 5 nodes in the network. Each Cluster member's node sends state message to its Cluster head. Cluster Head can sense data to the base station. Cluster Head always have maximum level of energy to charge its member nodes in the network.

\section{Sensing of data}

This phase, each node Send data to the base station through the neighbor nodes. Base station receives all the data via its neighbor node. Sensing of data is to reduce the energy of the node.

\section{Energy Estimation}

In this Phase, calculate the remaining energy of the node to update the energy level of the network. The energy level of the node in sensor network can be calculated after the data sensing to the base station. Here the minimum energy level of the node is set as 40 joule. All the nodes are boosted who is energy level comes under the minimum energy level. The sample energy level of the node in sensor network, after sensing data is given below:

\begin{tabular}{|c|c|}
\hline Node-Id & Remaining Energy \\
\hline 1 & 46 \\
\hline 2 & 49 \\
\hline 3 & 57 \\
\hline 4 & 61 \\
\hline 5 & 75 \\
\hline 6 & 30 \\
\hline 7 & 65 \\
\hline 8 & 82 \\
\hline 9 & 75 \\
\hline 10 & 41 \\
\hline 11 & 35 \\
\hline
\end{tabular}

\begin{tabular}{|c|c|}
\hline 12 & 69 \\
\hline 13 & 71 \\
\hline 14 & 76 \\
\hline 15 & 75 \\
\hline 16 & 36 \\
\hline 17 & 66 \\
\hline 18 & 50 \\
\hline 19 & 27 \\
\hline 20 & 32 \\
\hline 21 & 45 \\
\hline 22 & 30 \\
\hline 23 & 65 \\
\hline 24 & 40 \\
\hline 25 & 31 \\
\hline 26 & 42 \\
\hline 27 & 25 \\
\hline 28 & 34 \\
\hline 29 & 62 \\
\hline 30 & 69 \\
\hline 31 & 30 \\
\hline 32 & 41 \\
\hline 33 & 64 \\
\hline 34 & 57 \\
\hline 35 & 30 \\
\hline 36 & 47 \\
\hline
\end{tabular}

Table 1 Energy estimation

\section{Cluster Based Power Transfer}

This phase, including clustering concept. The Cluster head will recharge the cluster members when they are in need of energy boost up. Minimum threshold level is set. When nodes cross that level then notify to cluster head.

After Power transfer, node are update with following energy level.

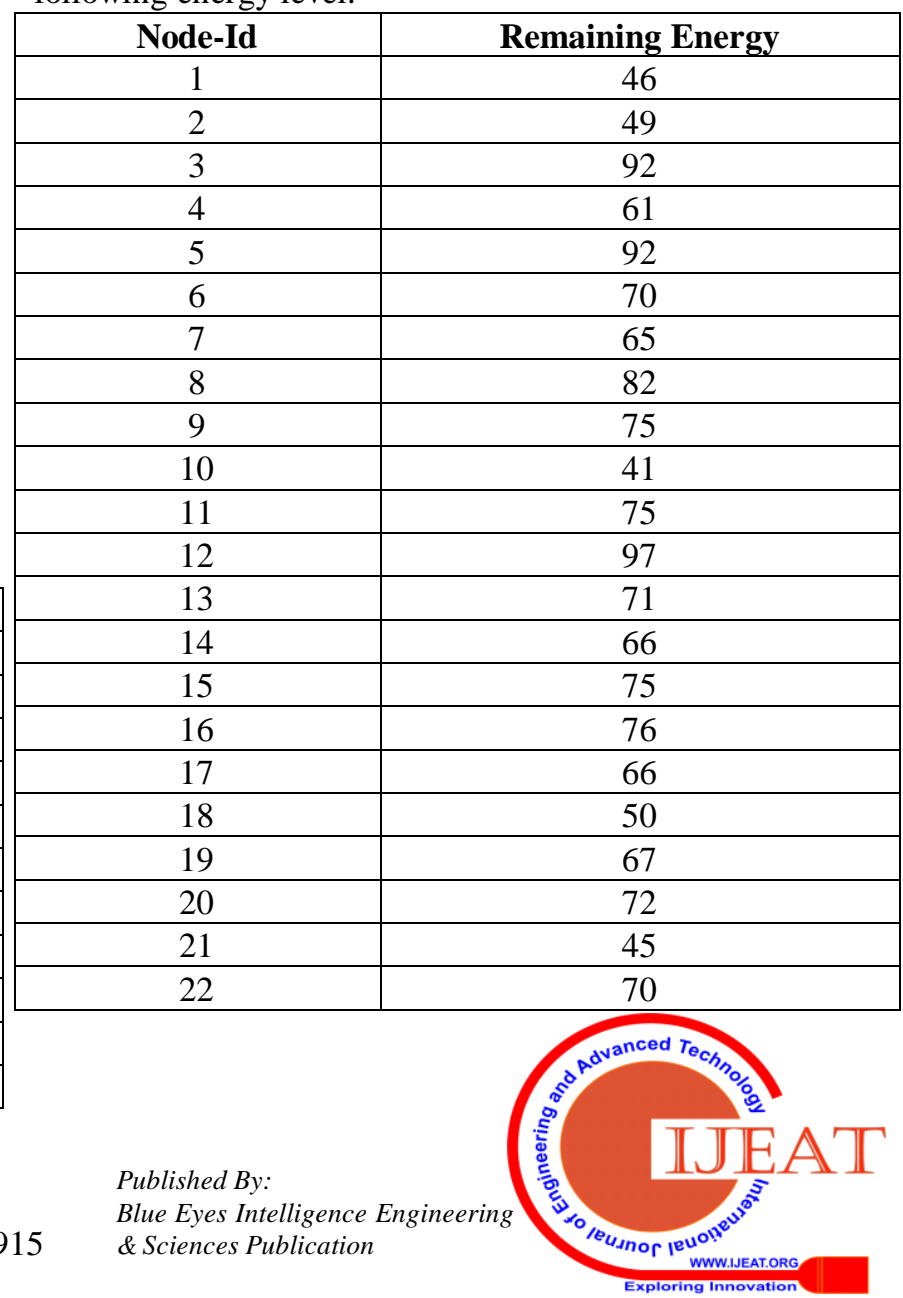




\begin{tabular}{|l|l|}
\hline 23 & 65 \\
\hline 24 & 40 \\
\hline 25 & 91 \\
\hline 26 & 42 \\
\hline 27 & 65 \\
\hline 28 & 74 \\
\hline 29 & 62 \\
\hline 30 & 69 \\
\hline 31 & 70 \\
\hline 32 & 41 \\
\hline 33 & 64 \\
\hline 34 & 57 \\
\hline 35 & 70 \\
\hline 36 & 47 \\
\hline
\end{tabular}

Table 2. Cluster Based Power Transfer

\section{METHODOLOGY}

\section{Energy Efficiency}

Vitality utilization is the most critical factor to decide the life of a sensor arrange in light of the fact that typically sensor hubs are driven by battery and have low vitality assets. This makes vitality improvement increasingly confused in sensor systems since it included decrease of vitality utilization as well as dragging out the life of the system however much as could be expected. This should be possible by having vitality mindfulness in each part of plan and task. This guarantees vitality mindfulness is likewise joined into gatherings of conveying sensor hubs and the whole system and not just in the individual hubs. A sensor hub more often than not comprises of four sub-frameworks

\section{Computing Subsystem}

It comprises of a microchip (microcontroller unit, $\mathrm{MCU}$ ) which is in charge of the control of the sensors and execution of correspondence conventions. MCU's typically work under different working modes for power the executives' purposes. Be that as it may, carrying between these working modes includes utilization of intensity, so the vitality utilization dimensions of the different modes ought to be considered while taking a gander at the battery lifetime of every hub.

\section{Communication Subsystem}

It comprises of a short range radio which is utilized to speak with neighboring hubs and the outside world. Radios can work under the Transmit, Receive, Idle and Sleep modes. It is essential to totally close down the radio as opposed to place it in the inactive mode when it isn't transmitting or getting in light of the high power expended in this mode.

\section{Sensing Subsystem}

It comprises of a gathering of sensors and actuators and connection the hub to the outside world. Vitality utilization can be decreased by utilizing low power segments and sparing force at the expense of execution which isn't required.

\section{Power Supply Subsystem}

It comprises of a battery which supplies capacity to the hub. It ought to be seen that the measure of intensity drawn from a battery is checked in such a case that high current is drawn from a battery for quite a while, the battery will bite the dust despite the fact that it could have continued for a more extended time. Normally the evaluated current limit of a battery being utilized for a sensor hub is lesser than the base vitality utilization expected prompting the lower battery lifetimes. The lifetime of a battery can be expanded by lessening the current definitely or notwithstanding turning it off regularly.

The power devoured by the sensor hubs can be decreased by creating structure strategies and models which help in vitality mindful plan of sensor systems. The lifetime of a sensor system can be expanded altogether if the working framework, the application layer and the system conventions are intended to be vitality mindful. Power the board in radios is vital in light of the fact that radio correspondence devours a great deal of vitality amid activity of the framework. Another part of sensor hubs is that a sensor hub additionally acts a switch and a lion's share of the bundles which the sensor gets are intended to be sent. Savvy radio equipment that assistance in recognizing and diverting parcels which should be sent and in the process diminish the figuring overhead on the grounds that the bundles are never again prepared in the middle of the road hubs.

Traffic can likewise be appropriated so as to boost the life of the system. A way ought not be utilized persistently to forward bundles paying little mind to how much vitality is spared in light of the fact that this drains the vitality of the hubs on this way and there is a break in the availability of the system. It is better that the heap of the traffic be conveyed all the more consistently all through the system.

\section{V.SYSTEM ARCHITECTURE}

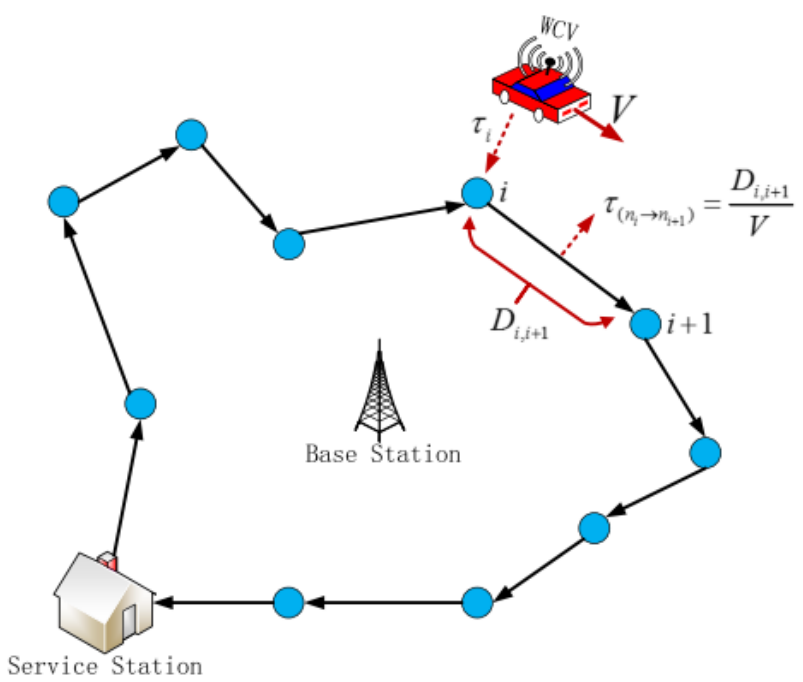

Figure 1. Architecture

\section{Algorithm}

Cluster Head will select based on the centrality concept (i.e.) the node have large number of neighbors will be selected as Cluster Head. In this method, the WCV (Wireless Charging Vehicle) need not to charge every node in the network. It will recharge only the Cluster Heads. These nodes (Cluster heads) will recharge the nodes which are in need of energy boost up within their transmission range. By using Clustering concept, the lifetime of the sensor 
network will improved.

The goal of the undertaking is to

- Improve the vitality productivity.

- Sensor hubs have constrained supply of vitality; subsequently, vitality proficiency is a noteworthy plan thought.

- Sensors lose their vitality while detecting.

- Energy productivity is a predominant thought regardless of what the issue is. This is on the grounds that sensor hubs just have a little and limited wellspring of vitality. Numerous arrangements, both equipment and programming related, have been proposed to improve vitality utilization.

\section{CONCLUSION}

This paper consider that a mobile wireless charging vehicle travels across the WSNs and charges nodes one by one to achieve a rechargeable wireless sensor network. For each node, the power of wireless charging varies from different wireless charging distances and angles. We exploit some effects of distance and dedicated vehicle for charging for efficiency in wireless charging. Our goal is to achieve an optimal cycle time over a cycle, thus the efficiency of wireless charging at each node should be maximized under restraints of distance and angle. We aim to find the optimal location for the WCV to charge nodes with maximum charging efficiency under the constraints of charging time.

\section{REFERENCE}

1. L. Anderegg and S. Eidenbenz, "Ad Hoc-VCG: A Truthful and Cost-Efficient Routing Protocol for Mobile Ad Hoc Networks with Selfish Agents,' in Proc. MobiCom, 2003, pp. 245-259.

2. S. Buchegger and J. Le Boudec, "Performance Analysis of the CONFIDANT Protocol,' ' in Proc. MobiHoc, 2002, pp. 226-236.

3. S. Buchegger and J. Le Boudec, "The Effect of Rumor Spreading in Reputation Systems for Mobile Ad-Hoc Networks,', in Proc. Workshop Model. Optim. Mobile, Ad Hoc Wireless Netw., 2003, pp. 131-140.

4. L. Buttya'n and J. Hubaux, "Enforcing Service Availability in Mobile Ad-Hoc WANs,'” in Proc. ACM MobiHoc, 2000, pp. 87-96.

5. L. Buttya'n and J. Hubaux, "Stimulating Cooperation in Self-Organizing Mobile Ad Hoc Networks,'” Mobile Netw. Appl., vol. 8, no. 5, pp. 579-592, Oct. 2003.

6. B. Edelman, M. Ostrovsky,M. Schwarz, T.D. Fudenberg, L. Kaplow, R. Lee, P. Milgrom, M.Niederle, and A. Pakes, "InternetAdvertising and the Generalized Second Price Auction: Selling Billions ofDollars Worth of Keywords,'” Amer. Econ. Rev., vol. 97, no. 1, pp. 242-259, Mar. 2005.

7. J. Feigenbaum, C. Papadimitriou, R. Sami, and S. Shenker, "A BGP-Based Mechanism for Lowest-Cost Routing,' Distrib. Comput. vol. 18 , no. 1, pp. 61-72, July 2005 .

8. J. Feigenbaum and S. Shenker, "Distributed Algorithmic Mechanism Design: Recent Results and Future Directions,", in Proc. 6th Int'lWorkshop Discr. Algorithms Methods Mobile Comput. Commun., 2002, pp. 1-13.

9. M. Feldman, J. Chuang, I. Stoica, and S. Shenker, "Hidden-Action in Multi-Hop Routing," in Proc. 6th ACM Conf. Electron. Commerce, 2005, pp. 117-126.

10. D. He, C. Chen, S. Chan, J. Bu, and P. Zhang, "Secure and Lightweight Network Admission and Transmission Protocol for Body Sensor Networks,' IEEE J. Biomed. Health Inf., vol. 17, no. 3, pp. 664-674, May 2013.

11. D. Karger and E. Nikolova, "On the Expected VCG Overpayment in Large Networks,' in Proc. 45th IEEE Conf. Decision Control, Dec. 2006, pp. 2831-2836.

12. L. Lamport, R. Shostak, and M. Pease, "The Byzantine Generals Problem,' ACM Trans. Programm. Lang. Syst., vol. 4, no. 3, pp. 382-401, July 1982.

13. Y. Li, "Toward a Qualitative Search Engine,' IEEE Internet Comput., vol. 2, no. 4, pp. 24-29, July/Aug. 1998.
14. S. Marti, T. Giuli, K. Lai, and M. Baker, "Mitigating Routing Misbehavior in Mobile Ad Hoc Networks," in Proc. ACM MobiCom, 2000, pp. 255-265.

15. P. Michiardi and R. Molva, "Core: A Collaborative Reputation Mechanism to Enforce Node Cooperation in Mobile Ad Hoc Networks,' in Proc. Adv. Commun. Multimedia Security, IFIP TC6/TC11 6th Joint Working Conf. Commun. Multimedia Security, 2002, pp. 107-121.

16. M. Osborne and A. Rubinstein, A Course in Game Theory. Cambridge, MA, USA: MIT Press, 1994.

17. A. Rodriguez-Mayol and J. Gozalvez, "Improving Selfishness Detection in Reputation Protocols for Cooperative Mobile Ad-Hoc Networks," in Proc. IEEE PIMRC, 2010, pp. 26-29.

18. X. Su, S. Chan, and G. Peng, "Auction in Multi-Path Multi-Hop Routing," IEEE Commun. Lett., vol. 13, no. 2, pp. 154-156, Feb. 2009.

19. X. Su, S. Chan, and G. Peng, "Generalized Second Price Auction in Multi-Path Routing with Selfish Nodes,' in Proc. IEEE GLOBECOM, 2009, pp. 3413-3418.

20. X. Su, G. Peng, and S. Chan, "FORBID: Cope with Byzantine Behaviors in Wireless Multi-Path Routing and Forwarding," in Proc. IEEE GLOBECOM, 2011, pp. 1-6.

21. K. Talwar, "The Price of Truth: Frugality in Truthful Mechanisms," in Proc. 20th Annu. Symp. Theor. Aspects Comput. Sci., 2003, pp. 608-619.

22. S. Tomasin, "Consensus-Based Detection of Malicious Nodes in Cooperative Wireless Networks,', IEEE Commun. Lett., vol. 15, no. 4, pp. 404-406, Apr. 2011.

23. H.R. Varian, “Position Auctions,'” Int'1 J. Ind. Org., vol. 25, no. 6, pp. 1163-1178, Dec. 2007

24. W. Wang, S. Eidenbenz, Y. Wang, and X.-Y. Li, "OURS: Optimal Unicast Routing Systems in Non-Cooperative Wireless Networks,' in Proc. MobiCom, 2006, pp. 402-413.

25. Y. Wang, V.C. Giruka, and M. Singhal, "Truthful Multipath Routing for Ad Hoc Networks With Selfish Nodes,' J. Parallel Distrib. Comput., vol. 68, no. 6, pp. 778-789, June 2008.

26. F. Wu, S. Zhong, and J. Liu, "Cost-Effective Traffic Assignment for Multipath Routing in Selfish Networks,' in Proc. IEEE GLOBECOM, 2007, pp. 453-457.

27. L. Yang, A. Cemerlic, and X. Cui, "A Dirichlet Reputation System in Reliable Routing of Wireless Ad Hoc Network,' Security Commun. Netw., vol. 3, no. 2/3, pp. 250-260, Mar.-June 2010.

28. S. Zhong, J. Chen, and Y. Yang, "Sprite: A Simple, Cheat-Proof, Credit-Based System for Mobile Ad-Hoc Networks,', in Proc. IEEE INFOCOM, 2003, pp. 1987-1997. 